\title{
Coulomb-Blockade Oscillations in Quantum Wires and Dots
}

H. van Houten, C.W.J. Beenakker, and A.A.M. Staring

Philips Research Laboratories, NL-5600 JA Eindhoven, The Netherlands

Abstract. Single electron tunneling can cause periodic oscillations to appear in the conductance as a function of gate voltage in split-gate nanostructures defined in the two-dimensional electron gas in GaAs-AlGaAs heterostructures. We review our theoretical and experimental results on these Coulomb-blockade oscillations in the resonant tunneling regime, where the transfer of single electrons proceeds through discrete energy levels.

\section{Introduction}

Oscillations in the conductance of a metallic grain as a function of the voltage on a gate electrode are a well-known manifestation of the Coulomb blockade of single electron tunneling [1,2]. The "orthodox" theory [3]-[5], supplemented by treatments of higher order tunneling processes and of the interaction of the tunneling electron with the electromagnetic environment [6]-[8], provide a detailed quantitative understanding of current experiments in metals [9]. Meanwhile, rapid progress has been made in the development of semiconductor nanostructures. The electron gas density in a two-dimensional electron gas (2DEG) in a Si MOSFET or in a GaAs-AlGaAs heterostructure is low, which is why the Fermi wavelength of the conduction electrons can be as large as 50 $\mathrm{nm}$ - two orders of magnitude larger than in metals. Lateral confinement of the electrons in the 2DEG on the scale of a few hundred nm, using split-gate techniques, has been exploited to make quantum wires and quantum dots [10].

After the discovery of conductance oscillations periodic in the gate voltage in a disordered quantum wire [11], and the identification of this phenomenon as Coulomb-blockade oscillations [12], it has become clear that in certain regimes single electron tunneling is the dominant transport mechanism in semiconductor nanostructures. This is exciting because of the possibility to study the interplay between size and charge quantization effects, which is not feasible in metallic grains (because of the small Fermi wavelength in a metal). This field has developed rapidly, as witnessed by the considerable number of recent experimental [13]-[22] and theoretical [23]-[26] papers.

Here we present an informal overview of our contributions. We discuss a theory for the period and the temperature dependence of the peak height of the Coulomb-blockade oscillations (Sec. 2). Experimental results on a quantum wire and a quantum dot are given in Sec. 3. We refer to a recent review article for a more complete discussion of single electron tunneling in semiconductor 


$$
U(N)=\frac{(N e)^{2}}{2 C}-N e \phi_{\mathrm{ext}}
$$

\section{Theory}

Consider the system of dot, leads, and gate electrode depicted in Fig. 1 (inset). The number $N$ of electrons on the dot may be treated as a sharply defined classical variable, provided the barriers have a tunnel resistance large compared to the resistance quantum $h / e^{2}$. The charging energy $U(N)$ of the dot may change discretely because of a change in the number $N$ of electrons on the dot (through tunneling to or from the leads), or it may change continuously because of a change in the voltage on the external gate electrode. We adopt the approximation of the orthodox model [4] of taking the charging energy into account macroscopically. We write $U(N)=\int_{0}^{-N c} \phi\left(Q^{\prime}\right) d Q^{\prime}$, where

$$
\phi(Q)=Q / C+\phi_{\text {ext }}
$$

is the electrostatic potential difference between dot and leads, $C$ is the mutual capacitance of the dot and its environment (gate plus leads), and $\phi_{\text {ext }}$ is an externally controlled potential proportional to the gate voltage (cf. Eq. (6) below). The charging energy then takes the form

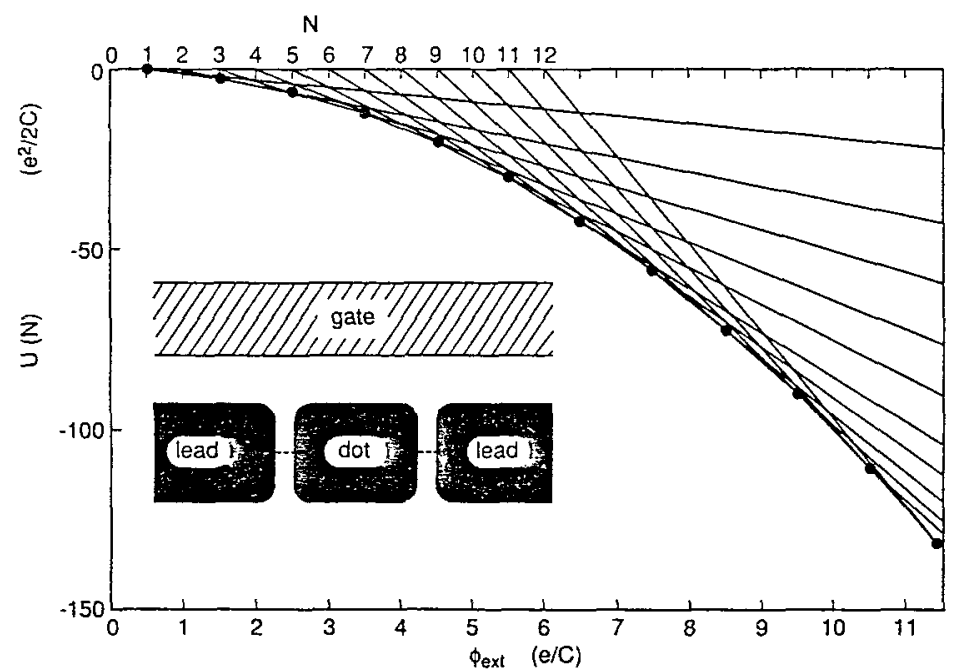

Figure 1: Charging energy of a dot, coupled by tunnel barriers to leads, as a function of the external electrostatic potential $\phi_{\text {ext }}$. Straight lines are according to Eq. (2) for a dot containing $N=1, \ldots, 12$ electrons. The ground state of the dot, for a given value of $\phi_{\text {ext }}$, corresponds to the minimum value of $U(N)$. Conductance peaks due to classical Coulomb-blockade oscillations occur if $U(N)=U(N-1)$ (full dots).
A metallic dot has a very small energy level separation at the Fermi level, so that the change in Fermi level by the transfer of a single electron from dot to leads may be neglected. The ground state of such a classical system corresponds to the minimum value of $U(N)$. The linear decrease of $U(N)$ with increasing $\phi_{\text {ext }}$ is illustrated in Fig. 1 for a set of values for $N$. Elastic tunneling, which requires the occupation sequence $N \rightarrow N-1 \rightarrow N \rightarrow N-1 \rightarrow \cdots$, is blocked at low temperatures, except when $U(N)=U(N-1)$, as indicated by the full dots in Fig. 1. The conductance thus exhibits peaks periodic in $\phi_{\text {ext }}$, located at

$$
\phi_{\mathrm{ext}}=\left(N-\frac{1}{2}\right) \frac{e}{C} .
$$

Eq. (3) does not apply to a quantum dot, in which the energy level separation can not be neglected. We denote by $E_{p}(p=1,2, \ldots)$ the single-electron energy levels in ascending order, measured relative to the bottom of the potential well in the quantum dot. Each level contains either one or zero electrons. Spin degeneracy, if present, can be included by counting each level twice, and other degeneracies can be included similarly. The energy levels $E_{p}$ depend on gate voltage and magnetic field, but are assumed to be independent of $N$. A peak in the low-temperature conductance now occurs whenever

$$
E_{N}+U(N)-U(N-1)=E_{\mathrm{F}}
$$

for some integer $N$. Eq. (4) equates the electrochemical potential in the dot (left hand side) to the Fermi level $E_{\mathrm{F}}$ in the leads (measured with respect to the local conduction band bottom). Substitution of Eq. (2) into Eq. (4) gives

$$
E_{N}+\left(N-\frac{1}{2}\right) \frac{e^{2}}{C}=E_{\mathrm{F}}+e \phi_{\mathrm{ext}}
$$

as the condition for a conductance peak [24]. If the incremental charging energy $e^{2} / C$ is much smaller than the level separation $\Delta E$, then Eq. (5) is the usual condition for resonant tunneling. In the opposite limit Eq. (3) is recovered.

In Fig. 2 we have illustrated the tunneling of an electron through the dot under the conditions of Eq. (5), with $N=9$. In panel (a) $N-1$ electrons occupy the dot. The $N$ th level in the dot is empty. In panel (b) an electron has tunneled into this level. The potential difference $\phi$ between dot and leads has decreased by $e / C$ (becoming negative), because of the added electron. Finally, in panel (c) the added electron tunmels out of the dot, resetting the potentials to the initial state of panel (a). In Fig. 2 the two leads are shown at the same to the initial state of panel (a). measurement a small voltage difference is imposed between the two leads. This voltage difference has no effect on the condition (5) if it is sufficiently small.

To determine the periodicity $\Delta \phi_{\text {gate }}$ of the conductance oscillations as a function of gate voltage we first need to know how $E_{\mathrm{F}}$ and the set of energy levels $E_{p}$ depend on the electrostatic potential from external charges $\phi_{\text {ext }}$. The 


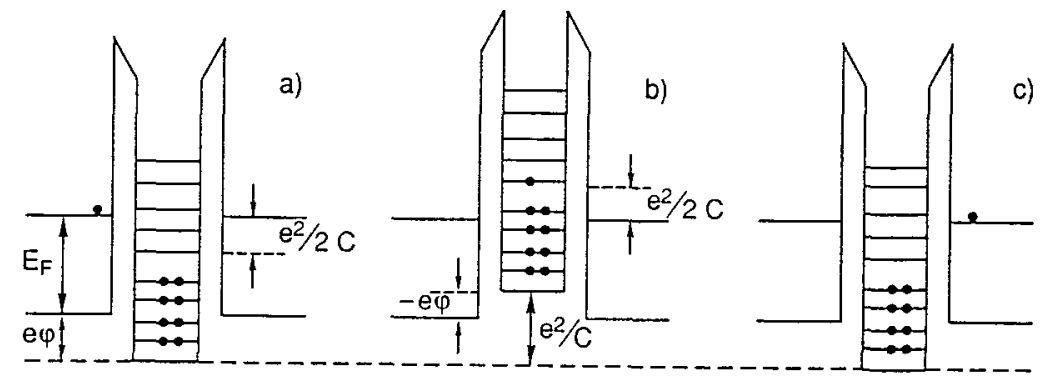

Figure 2: Single-electron tunneling through a quantum dot, under the conditions of Eq. (5), for the case that the charging energy is comparable to the level spacing. (From Ref. [24].)

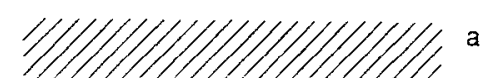

in

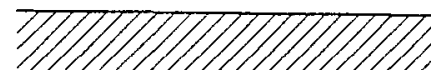

Figure 3: Schematic top-view of a quantum wire (a) and a quantum dot (b) Hatched regions denote gates on top of a GaAs-AlGaAs heterostructure containing a 2DEG. Non-depleted electron gas regions are black. Dashed lines indicate tunneling paths. (From Ref. [27].)

external charges are supplied by ionized donors and by a gate electrode. We denote by $\phi_{\text {gate }}$ the electrostatic potential difference between gate and 2DEG reservoir. One has

$$
\phi_{\text {ext }}=\phi_{\text {donors }}+\alpha \phi_{\text {gate }},
$$

with $\alpha$ a rational function of the capacitance matrix elements of the system. As illustrated in Fig. 3a, the periodic conductance oscillations in a quantum wire arise because of the natural formation of a conductance limiting segment when the wire is close to pinch-off. On average, the gate changes the density in the extended leads and the confined segment by an equal amount, because the density of states per unit area is the same. For equidistant energy levels in the confined segment we may then assume that $E_{\mathrm{F}}-E_{N}$ has the same value at each conductance peak. Eqs. (5) and (6) imply in this case a periodicity

$$
\Delta \phi_{\text {gate }}=\frac{e}{\alpha C} ; \quad \text { wire. }
$$

In the quantum dot geometry of Fig. $3 \mathrm{~b}$ the gates affect primarily the density in the dot, but not in the wide $2 D E G$ regions. In one period $E_{N}$ changes by an amount $\Delta E$, while $E_{\mathrm{F}}$ stays constant in this case. Eqs. (5) and (6) then imply

$$
\Delta \phi_{\text {gate }}=\frac{e}{\alpha C}\left(1+\frac{\Delta E}{e^{2} / C}\right) ; \quad \operatorname{dot} .
$$

The experimentally controlled variable is the gate voltage $V_{\text {gate }}$, which is the electrochemical (rather than electrostatic) potential difference between gate electrode and 2DEG reservoir. In the dot geometry (Fig. 3b) one has $\Delta V_{\text {gate }}=\Delta \phi_{\text {gate }}$, because the Fermi level (or chemical potential) of the 2DEG reservoirs is not affected by the gate. In the wire geometry (Fig. 3a) one has $\Delta V_{\text {gate }}=\Delta \phi_{\text {gate }}+\Delta E / \alpha e[27]$. From Eqs. (7) and (8) it then follows that in both geometries the periodicity in gate voltage is given by

$$
\begin{aligned}
\Delta V_{\text {gate }} & =\frac{e}{\alpha C}\left(1+\frac{\Delta E}{e^{2} / C}\right) \\
& \equiv \frac{e}{\alpha C_{\mathrm{T}}},
\end{aligned}
$$

where $C_{\mathrm{T}}$ is the thermodynamic capacitance (related to the electrostatic capacitance $C$ by $1 / C_{\mathrm{T}} \equiv 1 / C+\Delta E / e^{2}$ ). Since in the experiments $C$ is not known precisely, one can not easily infer the relative importance of size quantization effects from the periodicity alone. McEuen et al. [15] have circumvented this difficulty in an elegant experiment. They have been able to map out the energy level spectrum of a quantum dot in a strong magnetic field, by exploiting the fact that a magnetic field modulates $\Delta E$ and thus $C_{\mathrm{T}}$, but leaves $C$ unaffected.

Quantum size effects are more easily observed from the temperature dependence of the Coulomb-blockade oscillations. The temperature dependence of the peak height is shown in Fig. 4 for a quantum dot with equidistant energy levels, all with the same tunnel rates $\Gamma$ [24]. Also shown in Fig. 4 are the minima of the oscillations, which are seen to merge with the maxima as $k T$ approaches $e^{2} / C$. In the resonant tunneling regime $k T \lesssim \Delta E$ the peak height increases as the temperature is reduced, due to the diminished thermal broadening of the resonance. In the classical regime $e^{2} / C \gg k T \gg \Delta E$ there is a cancellation between the number of levels contributing to the conductance and the degree of thermal broadening, leading to a temperature-independent peak height [3]. At low temperatures the peak height saturates when $k T \lesssim h \Gamma$. The maximum peak height is $G_{\max }=e^{2} / h$ for the case of equal tunnel barriers. The lineshape in the low-temperature regime $k T \leqslant h \Gamma$ becomes asymmetric because of the Kondo effect $[28,29]$. This has not yet been observed experimentally.

The conductance minima $G_{\min }$ depend exponentially on the temperature, $G_{\min } \propto \exp \left(-E_{\text {act }} / k T\right)$, with activation energy [24]

$$
E_{\text {act }}=\frac{1}{2}\left(\Delta E+e^{2} / C\right) .
$$

This result holds for equal tunnel rates of the energy levels. The exponential decay of the conductance at the minima of the Coulomb blockade oscillations results from the suppression of tunneling processes which conserve energy in the intermediate state in the quantum dot. Tunneling via a virtual intermediate 


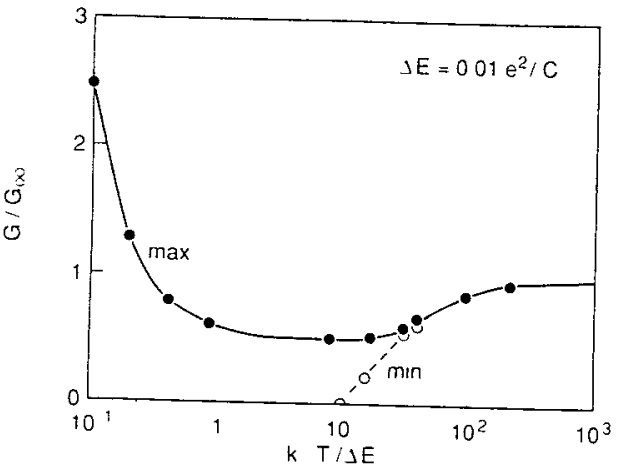

Figure 4: Temperature dependence of the maxima $(\max )$ and the minima ( $\mathrm{min}$ ) of the Coulomb-blockade oscillations, for a quantum dot with equidistant non-degenerate energy levels (at separation $\Delta E=0.01 e^{2} / C$ ), all with the same tunnel rates. The conductance is normalized to the high-temperature limit $G_{\infty} \equiv e^{2} \Gamma / 2 \Delta E$, which is the conductance of the two tunnel barriers added in series according to Ohm's law. (From Ref. [27].)

state is not suppressed at low temperatures, and may modify the temperature dependence of the minima if $h \Gamma$ is not much smaller than $k T$ and $\Delta E[6,7]$.

\section{$3 \quad$ Experiments}

In Fig. 5 the conductance versus gate voltage near pinch-off is shown for two disordered quantum wires, labeled D1 and D2 [16]. At $T=1.5 \mathrm{~K}$ both wires exhibit well-resolved conductance oscillations, which are periodic in the gate voltage. The oscillations are superimposed on a background conductance of approximately $0.1 e^{2} / h$, and have a period $\Delta V_{\text {gate }} \approx 2.7 \mathrm{mV}$ (D1) and 2.1 $\mathrm{mV}(\mathrm{D} 2)$. As the gate voltage is increased the oscillations disappear gradually. Whereas the two conductance traces are relatively similar at $T=1.5 \mathrm{~K}$, this is not the case at $T=50 \mathrm{mK}$. In wire $\mathrm{D} 2$ the oscillations become better resolved at this low temperature, while the period is unchanged and the value of the conductance at the maxima is also approximately the same. In contrast, the oscillations in wire D1 are suppressed at $50 \mathrm{mK}$, and an irregular pattern of sharp conductance peaks is observed instead. It is likely that this samplespecific effect reflects the presence of multiple segments in series in the quantum wire [16].

A magnetic field has no known effect on single electron tunneling in metals. In contrast, we find a rich variety of magnetic field effects in semiconductor nanostructures. For example, a field of $5 \mathrm{~T}$ was found to enhance the amplitude of the Coulomb-blockade oscillations in a quantum wire, whereas it reduced the aperiodic fluctuations seen at low temperatures [17]. A similar effect is seen in a quantum dot (Fig. 7, discussed below). Fig. 6a shows the temperature

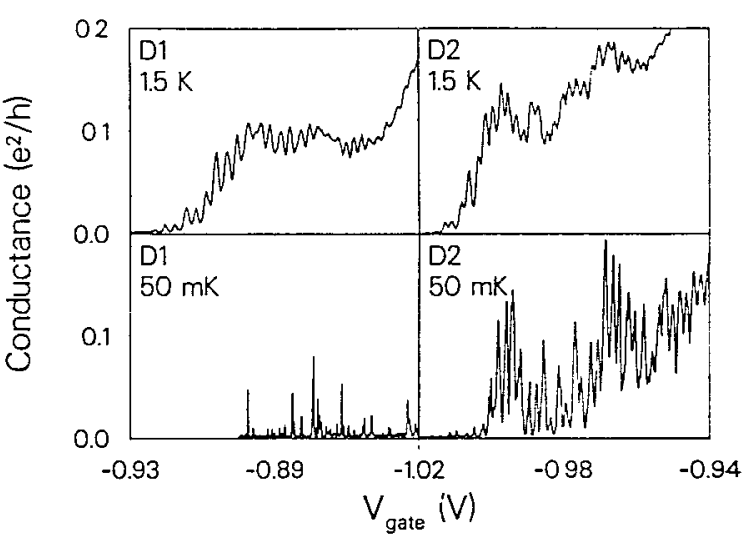

Figure 5: Two-terminal conductance versus gate voltage of two disordered quantum wires (D1 and D2) at $T=1.5 \mathrm{~K}$ and $50 \mathrm{mK}$. (From Ref. [16].)

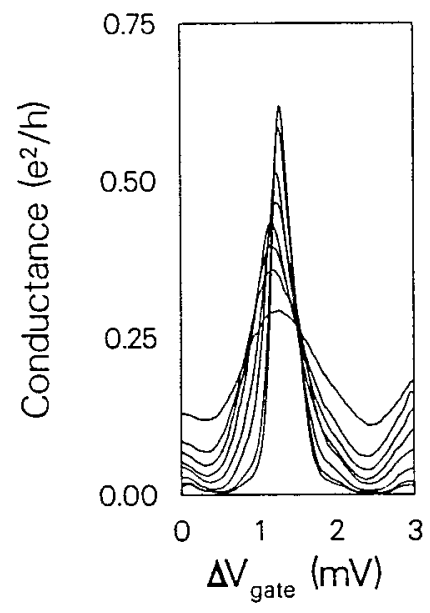

a)

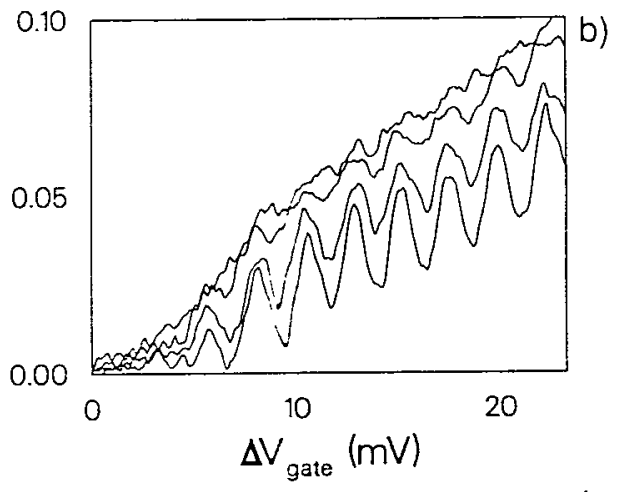

Figure 6: (a) Single conductance peak of a disordered quantum wire (D1) at $B=6.66 \mathrm{~T}$, for $T=110,190,290,380,490,590,710$, and $950 \mathrm{mK}$, from top to bottom. (b) Conductance oscillations versus gate voltage of a different disordered quantum wire for $T=3.2,2.5,1.6$, and $1 \mathrm{~K}$, from top to bottom. (From Ref. [16]).

dependence of one of the most pronounced peaks in the conductance of wire $\mathrm{D} 1$ for $B=6.66 \mathrm{~T}$. The peak height increases with decreasing temperature and reaches a value of $0.6 e^{2} / h$ at $T=100 \mathrm{mK}$. This behavior is characteristic of Coulomb-blockade oscillations in the resonant tunneling regime, as discussed in Sec. 2. The opposite temperature dependence is found at higher temperatures, as shown in Fig. 6b. This confirms the transition to the hightemperature classical regime where $k T$ exceeds the average level-spacing $\Delta E$ 


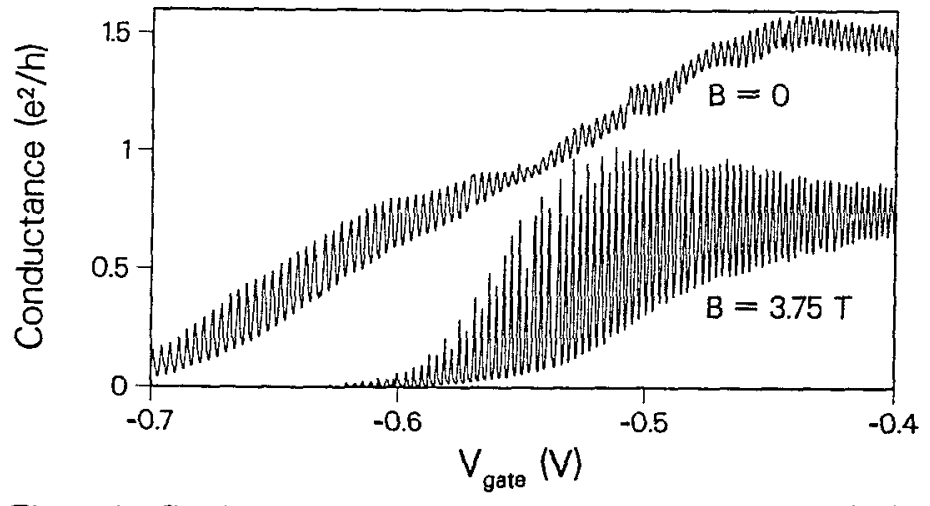

Figure 7: Conductance oscillations in a quantum dot in a GaAs-AlGaAs heterostructure, with a geometry as in Fig. 3b. The temperature is $100 \mathrm{mK}$. (From Ref. [17].)

of the conductance-limiting segment, and eventually approaches $e^{2} / C$ (cf. Fig. 4). A quantitative analysis of the period and temperature dependence of the conductance oscillations in our experiments on disordered quantum wires has shown that the regular oscillations can indeed be described consistently by the theory for Coulomb-blockade oscillations, in a regime where $e^{2} / C$ is larger than $\triangle E$ by about a factor of four $[16,27]$.

We now turn to a discussion of the conductance of a quantum dot of the design shown in Fig. 3b [17]. The device has two sets of gates to adjust the transmission probability of each tunnel barrier, and a third set to vary the potential $\phi_{\text {ext }}$ of the dot. (Because of the proximity of the gates the adjustments are not fully independent.) The tunnel barriers are formed by quantum point contacts close to pinch-off. Representative traces of conductance versus gate voltage at zero field and for $B=3.75 \mathrm{~T}$ are reproduced in Fig. 7. Quite spectacular oscillations are seen. They persist up to relatively high temperatures compared to the energy-level separation in the quantum dot, which confirms that they are Coulomb-blockade oscillations, rather than oscillations due to resonant tunneling of non-interacting electrons. The period of the oscillations and the maximum temperature at which they can be resolved are in reasonable agreement with theoretical estimates [17].

Our experiments on a quantum dot are done in a situation where the conductance of the individual barriers approaches $e^{2} / h$, which means that virtual tunneling processes have to be taken into account. Indeed, the conductance minima are not exponentially suppressed at $B=0$, see Fig. 7, even though the temperature was low $(100 \mathrm{mK})$. Experiments on a larger dot in the classical regime by Glattli et al. [22] confirm the importance of higher order tunneling processes. Unfortunately, these processes have not yet been studied theoretically for the quantum regime $k T<\Delta E$ of our experiment.

On application of an external magnetic field the background is suppressed, and the peak height is enhanced up to $e^{2} / h$. This effect is not yet understood.
The period is not changed, consistent with the analysis given in Sec. 2, for $e^{2} / C \gg \Delta E$.

In summary, semiconductor nanostructures present new possibilities for physicists interested in single electron tunneling. The period of the Coulombblockade oscillations is understood, as is the temperature dependence of the amplitude and lineshape in the resonant tunneling regime (as long as $k T>h \Gamma$ ). The transition to the ballistic regime, the role of virtual tunnelling processes, and the effects of a magnetic field remain to be further elucidated.

\section{Acknowledgements}

Part of the work described above has been done in collaboration with J. G. Williamson. GaAs-AlGaAs heterostructure material was provided by C. T. Foxon. We thank C. E. Timmering for technological work, S. Colak, L. P. Kouwenhoven, and N. C. van der Vaart for discussions, and J. H. Wolter and M. F. H. Schuurmans for support. This research was partly funded under the ESPRIT basic research action project 3133 .

\section{References}

[1] L. S. Kuz'min and K. K. Likharev, Pis'ma Zh. Eksp. Teor. Fiz. 45, 389 (1987) [JETP Lett. 45, 495 (1987)].

[2] T. A. Fulton and G. J. Dolan, Phys. Rev. Lett. 59, 109 (1987)

[3] R. I. Shekhter, Zh. Eksp. Teor. Fiz. 63, 1410 (1972) [Sov. Phys. JETP 36, 747 (1973)]; I. O. Kulik and R. I. Shekhter, Zh. Eksp. Teor. Fiz. 68, 623 (1975) [Sov. Phys. JETP 41, 308 (1975)].

[4] K. K. Likharev, IBM J. Res. Dev. 32, 144 (1988); D. V. Averin and K. K. Likharev, in: Mesoscopic Phenomena in Solids, B. L. Al'tshuler, P. A. Lee, and R. A. Webb, eds. (Elsevier, Amsterdam, 1991). This is a comprehensive review of single-electron tunneling in metals.

[5] L. I. Glazman and R. I. Shekhter, J. Phys. Condens. Matter 1, 5811 (1989).

[6] L. I. Glazman and K. A. Matveev, Pis'ma Zh. Eksp. Teor. Fiz. 48, 403 (1988) (JETP Lett. 48, 445 (1988)).

[7] D. V. Averin and A. A. Odintsov, Phys. Lett. A 140, 251 (1989); D. V. Averin and Yu. V. Nazarov, Phys. Rev. Lett. 65, 2446 (1990); A. A. Odintsov, G. Falci, and G. Schön, this volume.

[8] M. H. Devoret, D. Esteve, H. Grabert, G.-L. Ingold, H. Pothier, and C. Urbina, Phys. Rev. Lett. 64, 1824 (1990); G.-L. Ingold, this volume. 
D. V. A verin and A. N. Korotkov, Zh. Eksp. Teor. Fiz. 97, 1661 (1990) [Sov. Phys. JETP 70, 937 (1990)]; A. N. Korotkov, D. V. Averin, and K. K. Likharev, Physica B 165 \& 166, 927 (1990); D. V. Averin, A. N. Korotkov, and K. K. Likharev, submitted to Phys. Rev. B.

conduct Beenakker and H. van Houten, Quantum Transport in Semiconductor Nanostructures, Solid State Physics 44, 1 (1991).

[11] J. H. F. Scott-Thomas, S. B. Field, M. A. Kastner, H. I. Smith, and D. A. Antoniadis, Phys. Rev. Lett. 62, 583 (1989).

[24] C. W. J. Beenakker, Phys. Rev. B. 44, 1646 (1991); C. W. J. Beenakker, H. van Houten, and A. A. M. Staring, Phys. Rev. B. 44, 1657 (1991); and in: Granular Nanoelectronics, D. K. Ferry, J. Barker, and C. Jacoboni, eds. NATO ASI Series B 251 (Plenum, New York, 1991).

[13] S. B. Field, M. A. Kastner, U. Meirav, J. H. F. Scott-Thomas, D. A. Antoniadis, H. I. Smith, and S. J. Wind, Plyss. Rev. B 42, 3523 (1990).

[14] U. Meirav, M. A. Kastner, M. Heiblum, and S. J. Wind, Phys. Rev. B 40, 5871 (1989); U. Meirav, M. A. Kastner, and S. J. Wind, Phys. Rev. Lett. 65, 771 (1990).

[15] P. L. McEuen, E. B. Foxman, U. Meirav, M. A. Kastner, Y. Meir, N. S. Wingreen, and S. J. Wind, Phys. Rev. Lett. 66, 1926 (1991).

[16] A. A. M. Staring, H. van Houten, C. W. J. Beenakker, and C. T. Foxon, in: High Magnetic Fields in Semiconductor Physics III, G. Landwehr, ed. (Springer, Berlin, 1991); and submitted to Phys. Rev.
B.

[17] A. A. M. Staring, J. G. Williamson, H. van Houten, C. W. J. Beenakker, L. P. Kouwenhoven, and C. T. Foxon, Physica B, to be published; J. G. Williamson et al. Proc. Int. Symp. on Nanostructures and Mesoscopic Systems, W. P. Kirk, ed., to be published.

[18] L. P. Kouwenhoven, N. C. van der Vaart, A. T. Johnson, C. J. P. M. Harmans, J. G. Williamson, A. A. M. Staring, and C. T. Foxon, Festkörperprobleme/Advances in Solid State Physics 31, to be published; L. P. Kouwenhoven et al., Z. Phys. B, to be published. [19] C. de Graaf, J. Caro, S. Radelaar, V. Lauer, and K. Heyers, submitted
to Phys. Rev. B.

[20] R. J. Haug, K. Y. Lee, T. M. Smith, III, and J. M. Hong, in: Proc. 20th ICPS, E. M. Anastassakis and J. D. Joannopoulos, eds. (World Scientific, London, 1990); R. J. Haug, K. Y. Lee, and J. M. Hong this volume.

[21] C. J. B. Ford, Physica Scripta, to be published.

[22] D. C. Glattli, C. Pasquier, U. Meirav, F. I. B. Williams, Y. Jin, and B. Etienne, Z. Phys. B, to be published.

Y. Meir, N. S. Wingreen, and P. A. Lee, Phys. Rev. Lett. 66, 3048 (1991).

[26] A. Groshev, Phys. Rev. B 42, 5895 (1990); A. Groshev, T. Ivanov, and V. Valtchinov, Phys. Rev. Lett. 66, 1082 (1991).

[27] H. van Houten, C. W. J. Beenakker, and A. A. M. Staring, in: Single Charge Tunneling, H. Grabert and M. H. Devoret, eds., NATO ASI Series B (Plenum, New York), to be published.

[28] T. K. Ng and P. A. Lee, Phys. Rev. Lett. 61, 1768 (1988).

[29] L. I. Glazman and M. E. Raikh, Pis'ma Zh. Eksp. Teor. Fiz. 47, 378 (1988) (JETP Lett. 47, 452 (1988)). 
D. V. Averin and A. N. Korotkov, Zh. Eksp. Teor. Fiz. 97, 1661 (1990) [Sov. Phys. JETP 70, 937 (1990)]; A. N. Korotkov, D. V. Averin, and K. K. Likharev, Physica B 165 \& 166, 927 (1990); D. V. Averin, A. N. Korotkov, and K. K. Likharev, submitted to Phys. Rev. B.

[24] C. W. J. Beenakker, Phys. Rev. B. 44, 1646 (1991); C. W. J. Beenakker, H. van Houten, and A. A. M. Staring, Phys. Rev. B. 44, 1657 (1991); and in: Granular Nanoelectronics, D. K. Ferry, J. Barker, and C. Jacoboni, eds. NATO ASI Series B 251 (Plenum, New York, 1991).

[13] S. B. Field, M. A. Kastner, U. Meirav, J. H. F. Scott-Thomas, D. A. Antoniadis, H. I. Smith, and S. J. Wind, Phys. Rev. B 42, 3523 (1990).

[25] Y. Meir, N. S. Wingreen, and P. A. Lee, Phys. Rev. Lett. 66, 3048 (1991).

[26] A. Groshev, Phys. Rev. B 42, 5895 (1990); A. Groshev, T. Ivanov, and V. Valtchinov, Phys. Rev. Lett. 66, 1082 (1991).

[27] H. van Houten, C. W. J. Beenakker, and A. A. M. Staring, in: Single Charge Tunneling, H. Grabert and M. H. Devoret, eds., NATO ASI Series B (Plenum, New York), to be published.

[28] T. K. Ng and P. A. Lee, Phys. Rev. Lett. 61, 1768 (1988).

[29] L. I. Glazman and M. E. Raikh, Pis'ma Zh. Eksp. Teor. Fiz. 47, 378 (1988) (JETP Lett. 47, 452 (1988)).

[17] A. A. M. Staring, J. G. Williamson, H. van Houten, C. W. J. Beenakker, L. P. Kouwenhoven, and C. T. Foxon, Physica B, to be published; J. G. Williamson et al. Proc. Int. Symp. on Nanostructures and Mesoscopic Systems, W. P. Kirk, ed., to be published.

[18] L. P. Kouwenhoven, N. C. van der Vaart, A. T. Johnson, C. J. P. M. Harmans, J. G. Williamson, A. A. M. Staring, and C. T. Foxon, Festkörperprobleme/Advances in Solid State Physics 31, to be published; L. P. Kouwenhoven et al., Z. Phys. B, to be published. [19] C. de Graaf, J. Caro, S. Radeliar, V. Lauer, and K. Heyers, submitted
to Phys. Rev. B.

[20] R. J. Haug, K. Y. Lee, T. M. Smith, III, and J. M. Hong in: Proc 20th ICPS, E. M. Anastassakis and J. D. Joannopoulos, eds. (World Scientific, London, 1990); R. J. Haug, K. Y. Lee, and J. M. Hong,
this volume.

[21] C. J. B. Ford, Physica Scripta, to be published.

[22] D. C. Glattli, C. Pasquier, U. Meirav, F. I. B. Williams, Y. Jin, and B. Etienne, Z. Phys. B, to be published. 